\title{
Re-emergence of dengue virus serotype 3 infections in Gabon in 2016-2017, and evidence for the risk of repeated dengue virus infections
}

\author{
Haruka Abe $^{\mathrm{a}}$, Yuri Ushijima ${ }^{\mathrm{a}}$, Marguerite M. Loembe ${ }^{\mathrm{b}, \mathrm{c}}$, Rodrigue Bikangui ${ }^{\mathrm{b}, \mathrm{d}}$, \\ Georgelin Nguema-Ondo ${ }^{b}$, Patrick I. Mpingabo ${ }^{a}$, Vahid R. Zadeh ${ }^{a}$, Christelle M. Pemba ${ }^{a}$, \\ Yohei Kurosaki ${ }^{a}$, Yui Igasaki ${ }^{a}$, Sophia G. de Vries ${ }^{\text {b,e }}$, Martin P. Grobusch ${ }^{\text {b,d,e }}$, \\ Selidji T. Agnandji ${ }^{\mathrm{b}, \mathrm{d}}$, Bertrand Lell ${ }^{\mathrm{b}, \mathrm{d}, \mathrm{f}}$, Jiro Yasuda ${ }^{\mathrm{a}, \mathrm{g}, \mathrm{h}, *}$ \\ a Department of Emerging Infectious Diseases, Institute of Tropical Medicine (NEKKEN), Nagasaki University, Nagasaki, Japan \\ ${ }^{\mathrm{b}}$ Centre de Recherches Médicales de Lambaréné, Lambaréné, Gabon \\ ' Université des Sciences de la Santé de Libreville, Libreville, Gabon \\ 'Institute for Tropical Medicine, University of Tübingen, Tübingen, Germany \\ e Center of Tropical Medicine and Travel Medicine, Department of Infectious Diseases, Amsterdam University Medical Centers, Location AMC, University of \\ Amsterdam, Amsterdam, The Netherlands \\ ${ }^{\mathrm{f}}$ Division of Infectious Diseases and Tropical Medicine, Medical University of Vienna, Vienna, Austria \\ gational Research Center for the Control and Prevention of Infectious Diseases (CCPID), Nagasaki University, Nagasaki, Japan \\ ${ }^{\mathrm{h}}$ Graduate School of Biomedical Sciences, Nagasaki University, Nagasaki, Japan
}

\section{A R T I C L E IN F O}

\section{Article history:}

Received 12 October 2019

Received in revised form 1 December 2019

Accepted 3 December 2019

\section{Keywords:}

Dengue

Dengue virus

Gabon

Africa

Phylogeny

Surveillance

\begin{abstract}
A B S T R A C T
Objectives: Dengue outbreaks, mainly caused by dengue virus serotype 2 (DENV-2), occurred in 2007 and in 2010 in Gabon, Central Africa. However, information on DENV infections has been insufficient since 2010. The aim of this study was to investigate the current DENV infection scenario and the risk of repeated infections in Gabon.

Methods: During 2015-2017, serum samples were collected from enrolled febrile participants and were tested for DENV infection using RT-qPCR. DENV-positive samples were analyzed for a history of previous DENV infection(s) using ELISA. The complete DENV genome was sequenced to analyze the phylogeny of Gabonese DENV strains.

Results: DENV-3 was exclusively detected, with a high rate of anti-DENV IgG seropositivity among DENV3-positive participants. DENV-3 showed higher infection rates in adults and the infection was seasonal with peaks in the rainy seasons. Phylogenetic analysis revealed that Gabonese DENV-3 originated from West African strains and has been circulating continuously in Gabon since at least 2010, when the first DENV-3 case was reported.

Conclusions: These findings indicate stable DENV-3 circulation and the risk of repeated DENV infections in Gabon, highlighting the need for continuous monitoring to control DENV infections.

(c) 2019 The Authors. Published by Elsevier Ltd on behalf of International Society for Infectious Diseases. This is an open access article under the CC BY-NC-ND license (http://creativecommons.org/licenses/by-
\end{abstract} nc-nd/4.0/).

\section{Introduction}

With a significant increase in incidence in the past decades, dengue has become one of the most prevalent infectious diseases and a major health concern in tropical and subtropical regions of the

\footnotetext{
* Corresponding author at: Department of Emerging Infectious Diseases, Institute of Tropical Medicine, Nagasaki University, 1-12-4 Sakamoto, Nagasaki 852-8523, Japan.

E-mail address: j-yasuda@nagasaki-u.ac.jp (J. Yasuda).
}

world, including more than 100 countries in the Southeast Asia, Africa, North and South America, and Western Pacific regions (Brady et al., 2012). Dengue virus (DENV) is estimated to cause 390 million infections annually, and 3.6 billion people are at risk of DENV infection (Bhatt et al., 2013). The incidence of dengue increased seven-fold between 1990 and 2013, with 8.9 billion dollars of global economic burden for dengue in 2013 (Shepard et al., 2016).

DENV is mainly transmitted by the mosquito vectors Aedes aegypti and Aedes albopictus and has four serotypes: DENV serotypes 1-4 (DENV-1 to DENV-4) (Simmons et al., 2012). In Africa, DENV infections have been reported in at least 34 countries, 
where DENV-1 and DENV-2 are the main causes of dengue; however, it is quite likely that DENV infection cases have been underreported due to a prevailing clinical picture of undifferentiated fever and the unavailability of appropriate diagnostic tests in some settings (Amarasinghe et al., 2011). Currently, there is a very limited number of reports regarding DENV-3 and DENV-4 infections in Africa; whole-genome information is available for only two African DENV-3 strains (Mozambique, 1985, GenBank accession number FJ882575; Senegal, 2009, GenBank accession number KU509282) and is still unavailable for African DENV-4.

In Gabon, a Central African country, two dengue outbreaks were reported in 2007 and 2010 (Caron et al., 2012). DENV-2 was mainly detected on both occasions and this serotype caused nearly $95 \%$ of DENV infections. DENV-1 caused a small number of infections in the 2010 outbreak. In contrast, DENV-3 has only been detected in one individual in Gabon so far (Caron et al., 2013). Since the 2010 outbreak, there has been little information on DENV infections in the country, with no evidence of DENV circulation across rural Gabon, as reported in 2011 (Pourrut et al., 2011). However, in the area around Lambaréné, an anti-DENV immunoglobulin $G(\operatorname{IgG})$ seropositivity rate of $12.3 \%$ was found in an analysis of the sera from 30-month-old children that was collected before the 2007 and 2010 outbreaks; this indicated a pre-outbreak circulation of DENV (Gabor et al., 2016). These data on DENV prevalence seem to be contradictory, requiring further surveillance studies to clarify the current scenario of both DENV circulation in Gabon and the anti-DENV IgG seropositivity rate, which depicts repeated DENV infection in febrile patients.

As part of a project towards establishing a surveillance system for DENV infections in Lambaréné, Gabon, we conducted serum sample collection from febrile patients to investigate current DENV infections and the genetic characteristics of DENV strains circulating in Gabon. This showed evidence of endemic DENV-3 circulation that has been maintained since the first DENV-3 detection in 2010 in Gabon. A high rate of anti-DENV IgG seropositivity among DENV-3-positive patients was also found, indicating that the residents around Lambaréné are facing the risk of repeated DENV infections.

\section{Materials and methods}

\section{Study population}

In this study, the samples were collected at two medical facilities, the Albert Schweitzer Hospital in Lambaréné and the Centre de Recherches Médicales de Lambaréné (CERMEL). The Albert Schweitzer Hospital is one of the main general hospitals in the study area, and CERMEL is the medical institute that has a clinical department. Febrile patients (body temperature $\geq 37.5^{\circ} \mathrm{C}$ ) who visited the two medical facilities between January 2015 and June 2017 were recruited for enrolment in a surveillance study on DENV infections. In this study, the age of the participants was restricted to $>1$ year. Demographic information (age and sex) of the recruited participants was collected.

\section{Ethics statement}

This study was approved by the institutional review boards of CERMEL and Nagasaki University (approval numbers CEI-007 and 170921177, respectively). Written informed consent was obtained from all of the participants or their parents.

Viral RNA extraction and detection by reverse transcriptionquantitative polymerase chain reaction ( $R T-q P C R$ )

Viral RNA was extracted from $140 \mu$ l of each serum sample with a QIAamp Viral RNA Mini Kit (Qiagen, Hilden, Germany) according to the manufacturer's instructions. RT-qPCR was performed in a $20-\mu l$ reaction using a QuantiTect Probe RT-PCR Kit (Qiagen). Each reaction mixture contained $10 \mu \mathrm{l} 2 \times$ QuantiTect Probe RT-PCR Master Mix, $0.2 \mu \mathrm{l}$ QuantiTect RT Mix, $0.5 \mu \mathrm{M}$ of each primer, $0.2 \mu \mathrm{M}$ TaqMan probe, $2 \mu \mathrm{l}$ RNA template, and RNase-free water up to $20 \mu \mathrm{l}$. RT-qPCR assays were conducted using a LightCycler 480 instrument (Roche, Basel, Switzerland) under the following conditions: $30 \mathrm{~min}$ at $50^{\circ} \mathrm{C} ; 15 \mathrm{~min}$ at $95^{\circ} \mathrm{C}$; and 45 cycles of 15 $\mathrm{s}$ at $95^{\circ} \mathrm{C}$, and $60 \mathrm{~s}$ at $60^{\circ} \mathrm{C}$. The primers and the probe were designed using the sequences reported previously (Santiago et al., 2013). Data from the RT-qPCR assays were analyzed using software included in the LightCycler 480 system. RT-qPCR assays were performed in duplicate and the samples showing cycle threshold $(\mathrm{Ct})$ values under 40 were set as positive.

\section{Enzyme-linked immunosorbent assay (ELISA) of anti-DENV IgG antibodies}

Anti-DENV IgG antibodies were detected using the Dengue Virus IgG DxSelect Kit (Focus Diagnostics, Cypress, CA, USA) and the signal intensities were normalized by the calibrator value according to the manufacturer's protocol. The normalized signals that displayed $>1.0$ were defined as positive. A correlation between the $\mathrm{Ct}$ values of RT-qPCR and the normalized signal intensities of ELISA was calculated using GraphPad Prism 7 software (GraphPad Software, San Diego, CA, USA).

\section{Sequencing of the envelope gene for genotyping of detected DENV} strains

Amplification of the full-length envelope gene (1479bp) of DENV-3 strains was performed with the PrimeScript II High Fidelity One Step RT-PCR Kit (Takara Bio, Shiga, Japan) using primers reported previously (Shihada et al., 2017). After agarose gel purification was performed with a QIAquick Gel Extraction Kit (Qiagen), the PCR products were processed using the BigDye Terminator v3.1 Cycle Sequencing Kit (Thermo Fisher Scientific, Waltham, MA, USA) and analyzed using an ABI3500 capillary sequencer (Thermo Fisher Scientific) to obtain sequence data. The genotypes of DENV strains were determined through BLAST analysis of the sequence data (https://blast.ncbi.nlm.nih.gov/ Blast.cgi).

\section{Whole-genome sequencing}

Libraries were prepared from extracted viral RNA samples using a TruSeq RNA Library Preparation Kit v2 (Illumina, San Diego, CA, USA) according to the manufacturer's instructions. After quality and quantity check of each library using an Agilent 2100 Bioanalyzer (Agilent Technologies, Santa Clara, CA, USA) with a High Sensitivity DNA Kit (Agilent), sequencing was performed using a 300-cycle High Output Kit (Illumina) on a MiniSeq sequencer (Illumina). Mapping of the paired-end reads was performed on CLC Genomics Workbench 11.0.1 software (Qiagen) using the whole-genome sequence of the Senegal 2009 strain as a template. Consensus sequences were extracted and aligned with reference strains on BioEdit 7.0.5.3 software (http://www.mbio. ncsu.edu/BioEdit/bioedit.html).

\section{Phylogenetic analysis}

To infer the phylogeny of full-length envelope gene sequences of DENV-3 strains, a Bayesian analysis was performed with timestamped reference sequences that include all complete envelope gene sequences of African DENV-3 strains using BEAST v1.8.0 software (http://beast.community/) under the conditions of the 
Table 1

Demographic and laboratory data of patients infected with DENV-3.

\begin{tabular}{|c|c|c|c|c|c|}
\hline Sample ID & $\begin{array}{l}\text { Age } \\
\text { (years) }\end{array}$ & Sex & Ct value ${ }^{a}$ & IgG signal $^{\mathrm{b}}$ & Collection Date \\
\hline 01 & 19 & $\mathrm{M}$ & 24.19 & +++ & 9 May 2016 \\
\hline 02 & 33 & $\mathrm{~F}$ & 20.24 & + & 17 May 2016 \\
\hline 03 & 28 & $\mathrm{M}$ & 18.55 & +++ & 2 Jun 2016 \\
\hline 04 & 46 & $\mathrm{~F}$ & 30.33 & +++ & 17 Jun 2016 \\
\hline 05 & 18 & $\mathrm{~F}$ & 34.84 & +++ & 21 Jun 2016 \\
\hline 06 & 43 & $\mathrm{~F}$ & 34.55 & +++ & $05 \mathrm{Jul} 2016$ \\
\hline 07 & 36 & $\mathrm{~F}$ & 23.95 & +++ & 14 Jul 2016 \\
\hline 08 & 4 & M & 33.91 & ++ & 15 Jul 2016 \\
\hline 09 & 29 & $\mathrm{~F}$ & 18.32 & - & 22 Jul 2016 \\
\hline 10 & 6 & $\mathrm{~F}$ & 30.08 & + & 08 Aug 2016 \\
\hline 11 & 26 & $\mathrm{M}$ & 34.54 & +++ & 22 Aug 2016 \\
\hline 12 & 26 & $\mathrm{~F}$ & 35.11 & - & 28 Sep 2016 \\
\hline 13 & 21 & M & 36.79 & +++ & 08 Oct 2016 \\
\hline 14 & 44 & M & 36.81 & +++ & 10 Nov 2016 \\
\hline 15 & 29 & $\mathrm{M}$ & 33.99 & +++ & 05 Dec 2016 \\
\hline 16 & 24 & $\mathrm{~F}$ & 33.00 & +++ & 10 May 2017 \\
\hline 17 & 57 & M & 27.78 & +++ & 15 May 2017 \\
\hline
\end{tabular}

DENV-3, dengue virus serotype 3; $\mathrm{M}$, male; $\mathrm{F}$, female.

a Cycle threshold $(\mathrm{Ct})$ value of virus detection by RT-qPCR.

b Signal intensities of ELISA tests for anti-DENV IgG are presented as normalized signal intensities: -, signal <1.0 (negative); +, signal 1.0-3.0; ++, signal 3.1-5.0; +++, signal $>5.0$.

SRD08 model, the relaxed lognormal clock, and 80 million generations. For countries outside Africa, reference strains were widely selected from each continent and from various time points of the collection date. The effective sample size (ESS) values were verified to show more than 1000 . For complete genome sequences of DENV-3 strains, all whole-genome information of reference strains was obtained from each continent and from various time points of the collection date, as described above. Consensus sequences of DENV-3 strains detected in this study and reference sequences were aligned and analyzed using a maximumlikelihood method with a general time reversible model and gamma distribution of substitution rate. A total of 1000 bootstrap replicates were generated in this analysis. Phylogenetic trees were visualized and modified using FigTree v1.4.2 software (http://tree. bio.ed.ac.uk/software/figtree).

\section{Statistical analysis}

The statistical data analysis was performed using GraphPad Prism 7 software (GraphPad Software, San Diego, CA, USA). The Chi-square test or Fisher's exact test was used to determine the significance of differences in the comparisons of general categorical variables. Results were considered to be statistically significant when the $p$-value was less than 0.05 .

\section{Sequence data availability}

The sequences of the DENV-3 strains obtained in this study have been deposited in GenBank with accession numbers of LC379193LC379202.

\section{Results}

\section{Study population and DENV detection}

During 2015-2017, 1007 serum samples were collected from febrile patients (body temperature $>37.5^{\circ} \mathrm{C}$ ) who were enrolled in this study. The age range was $1-82$ years and the mean age was $10.83 \pm 14.4$ years ( \pm standard deviation). The samples were obtained from 186 adult patients ( $\geq 18$ years of age) and 805 pediatric patients ( $<18$ years of age); no age information was available for 16 samples. Samples were screened for DENV (serotypes 1-4) by RT-qPCR, as described previously (Santiago et al., 2013). Overall, 17 samples were found to be positive for DENV-3 (Table 1), including 15 samples from adult patients (positivity rate in adults, $8.06 \%$ ) and two from pediatric patients (positivity rate in children, $0.25 \%$ ) (Table 1 ). Comparison of the mean age for all samples showed significant differences between the DENV-3-positive and negative populations (Table 2). However, there were no statistically significant differences in DENV-3 positivity rates in each of the adult or pediatric population, and between the female and male populations (Table 3 ).

As serum samples were collected continuously from January 2015 to June 2017 (Figure 1A), the seasonality of DENV-3 infections was analyzed. Considering that most of the DENV-3-positive samples were derived from adult patients, the seasonal change in the DENV-3 positivity rate was analyzed in the adult population. The result showed peaks of DENV-3 infections in May and September-October, during the rainy season (Figure 1B).

\section{Detection of anti-DENV IgG antibodies in DENV-3-positive patients}

To screen DENV-positive patients for potential previous DENV infection(s), an ELISA test was performed against anti-DENV IgG antibodies. The ELISA results showed that most of the DENVpositive patients identified by RT-qPCR were also positive for antiDENV IgG, with a positivity rate of $88.24 \%$ (Table 1 ). To investigate the suppression of viral replication by IgG production in the febrile phase of patients who had experienced a previous DENV infection (s), correlation was analyzed between Ct values of RT-qPCR and the

Table 2

Comparison of age between the patients who were positive for DENV-3 and those who were negative for DENV-3.

\begin{tabular}{|c|c|c|c|c|c|c|}
\hline & \multirow[b]{2}{*}{ Sex } & \multicolumn{2}{|l|}{ Negative } & \multicolumn{2}{|l|}{ Positive } & \multirow[t]{2}{*}{$p$-Value } \\
\hline & & Age (years) ${ }^{\mathrm{a}}$ & $(95 \% \mathrm{CI})$ & Age (years) & $(95 \% \mathrm{CI})$ & \\
\hline \multirow[t]{3}{*}{ All patients } & Mix & 10.38 & $(9.50-11.26)$ & 28.76 & $(21.68-35.85)$ & $<0.001$ \\
\hline & $\mathrm{F}$ & 10.80 & $(9.48-12.13)$ & 29.00 & $(19.45-38.55)$ & $<0.001$ \\
\hline & M & 9.95 & $(8.77-11.14)$ & 28.50 & $(15.07-41.93)$ & $<0.001$ \\
\hline \multirow[t]{3}{*}{ Adults ( $\geq 18$ years) } & Mix & 36.42 & $(34.08-38.75)$ & 31.93 & $(25.73-38.14)$ & 0.27 \\
\hline & $\mathrm{F}$ & 36.91 & $(33.57-40.25)$ & 31.88 & $(23.89-39.86)$ & 0.38 \\
\hline & M & 36.02 & $(32.68-39.37)$ & 32.00 & $(19.36-44.64)$ & 0.50 \\
\hline \multirow[t]{3}{*}{ Pediatrics (<18 years) } & Mix & 4.84 & $(4.59-5.09)$ & 5.00 & $(-7.71-17.71)$ & 0.95 \\
\hline & $\mathrm{F}$ & 4.77 & $(4.42-5.12)$ & 6.00 & $\mathrm{~N} / \mathrm{A}^{\mathrm{b}}$ & $\mathrm{N} / \mathrm{A}$ \\
\hline & M & 4.90 & $(4.54-5.26)$ & 4.00 & $\mathrm{~N} / \mathrm{A}$ & $\mathrm{N} / \mathrm{A}$ \\
\hline
\end{tabular}

DENV-3, dengue virus serotype 3; F, female; $\mathrm{M}$, male; 95\% CI, 95\% confidence interval; N/A, not analyzed.

a Age is presented as mean years.

b DENV-3 positive numbers in pediatric patients were too small to analyze statistically. 
Table 3

Comparison of sex between the patients who were positive for DENV-3 and those who were negative for DENV-3.

\begin{tabular}{llllll}
\hline & Sex & Negative $^{\mathrm{a}}$ & Positive & OR & $p$-Value \\
\hline All patients & F & 475 & 9 & 1.19 & 0.72 \\
& M & 502 & 8 & - & - \\
Adults ( $\geq 18$ years $)$ & F & 84 & 8 & 1.07 & 0.90 \\
\multirow{2}{*}{ Pediatrics (<18 years) } & M & 90 & 7 & - & - \\
& F & 385 & 1 & 1.09 & $>0.99$ \\
& M & 418 & 1 & - & - \\
\hline
\end{tabular}

DENV-3, dengue virus serotype 3; OR, odds ratio; F, female; $\mathrm{M}$, male.

a Thirteen DENV-3 negative patients were excluded from the analysis due to the lack of sex information.

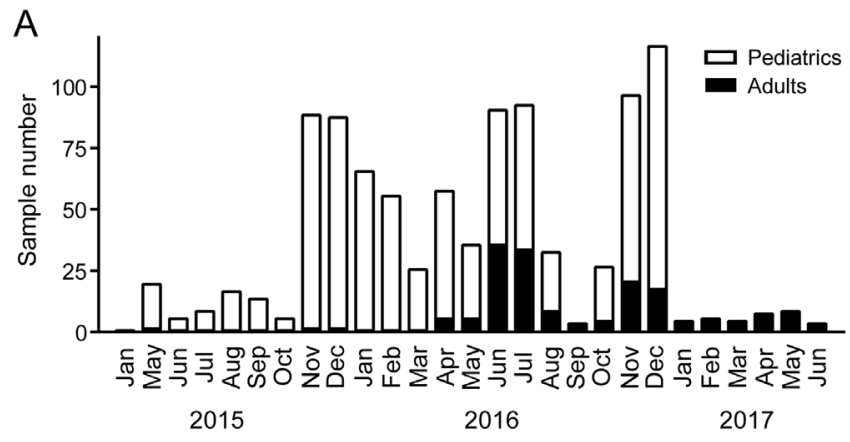

B

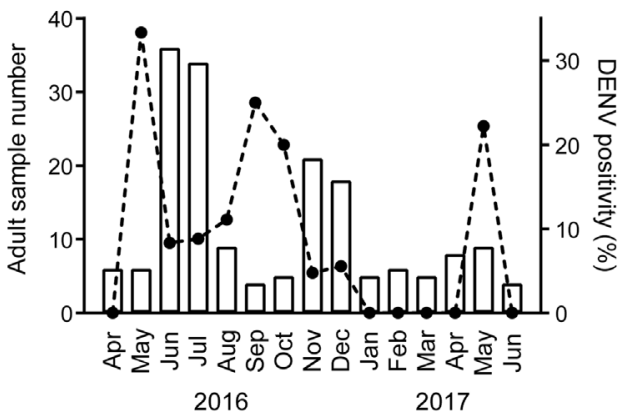

Figure 1. Seasonal data of sample collection and DENV positivity. (A) Sample numbers collected from febrile patients during the study. White and black columns show pediatric and adult sample numbers, respectively. (B) DENV positivity values in adults from April 2016 to June 2017. Columns and the dotted line depict the adult sample number and DENV positivity, respectively.

signal intensities of ELISA tests. Although there were two ELISAnegative samples, Ct values were significantly correlated with ELISA signal intensities, indicative of effective blockade of viral replication by rapid IgG production (Figure 2 ).

\section{Phylogenetic analysis of detected DENV-3 strains}

To investigate the genetic characteristics of DENV-3 strains detected in this study, sequences of the full-length envelope glycoprotein gene were obtained from 10 samples (eight samples from adults and two from pediatric participants, with GenBank accession numbers LC379193-LC379202), using a method described previously (Shihada et al., 2017). Phylogenetic analysis

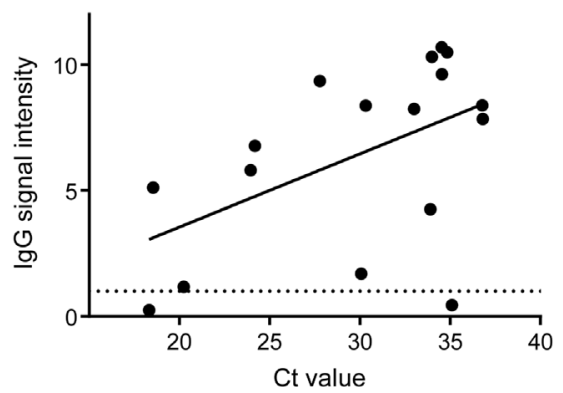

Figure 2. Correlation between the cycle threshold $(\mathrm{Ct})$ values of RT-qPCR and signal intensities of anti-DENV IgG as analyzed by ELISA. Pearson's correlation coefficient and significance were calculated using GraphPad Prism 7 software $(n=17, p<0.05$, $r=0.499$ ). The dotted line depicts the threshold to determine ELISA positivity (signal intensity $=1.0$ )

inferred that the detected DENV-3 strains belonged to the African cluster of genotype III (Figure 3). The Gabonese DENV-3 strain detected in 2010 (GenBank accession number JX080299), the only DENV-3 strain that has been reported so far in Gabon, was genetically the closest to the strains detected in this study, with a sequence homology that ranged between $99.47 \%$ and $99.79 \%$. In spite of the existence of some other African strains detected after 2010 (e.g. Djibouti 2012, KT187294; West Africa-Germany 2013, KJ922394), these post-2010 African strains were all located outside of the Gabonese cluster, indicating that DENV-3 was stably maintained in Gabon (Figure 3).

It was attempted to sequence the complete genome of the DENV-3 strains detected in this study by next-generation sequencing. Complete genome sequences of DENV-3 strains were obtained from five samples collected from adult patients (GenBank accession numbers LC379193-LC379197). Phylogenetic analysis was performed with whole-genome sequences including two strains that constitute all African DENV-3 complete sequences available (Mozambique, 1985 and Senegal, 2009). The result indicated that the Senegal 2009 strain is genetically close to the DENV-3 strains sequenced in this study (Figure 4). Interestingly, a few Chinese strains detected in 2009 were also inferred as the genetically closest strains, with no relationships to other Chinese strains detected in different years. The amino acid sequence homology between the DENV-3 strains detected in this study and the Senegal 2009 strain was 99.00-99.16\%. The amino acid substitutions tended to be located in non-structural proteins, especially in the NS5 protein (Table 4). These substitutions, however, have not yet been reported to correlate with the replication and pathogenicity of DENV.

\section{Discussion}

There were two dengue outbreaks in Gabon in 2007 and 2010, which were caused by DENV-2 in the vast majority of cases and DENV-1 in a small number of patients (Caron et al., 2013). In this study, DENV-3 was exclusively detected in febrile patients during the years 2016 and 2017, possibly indicative of serotype shift on mainly circulating DENV in Gabon, as shown previously in a report of longitudinal surveillance (Nisalak et al., 2003). As recognized generally (WHO, 2019), seasonal changes in DENV-3 positivity rate with peaks in the rainy seasons were also observed, probably reflecting an increase in mosquito vector activity.

A meta-analysis study suggested the possibility of DENV-3 infections being significantly related to severe cases (Soo et al., 2016), raising the alert for the careful continuous monitoring of the dissemination of DENV-3 infections. So far, limited cases of 


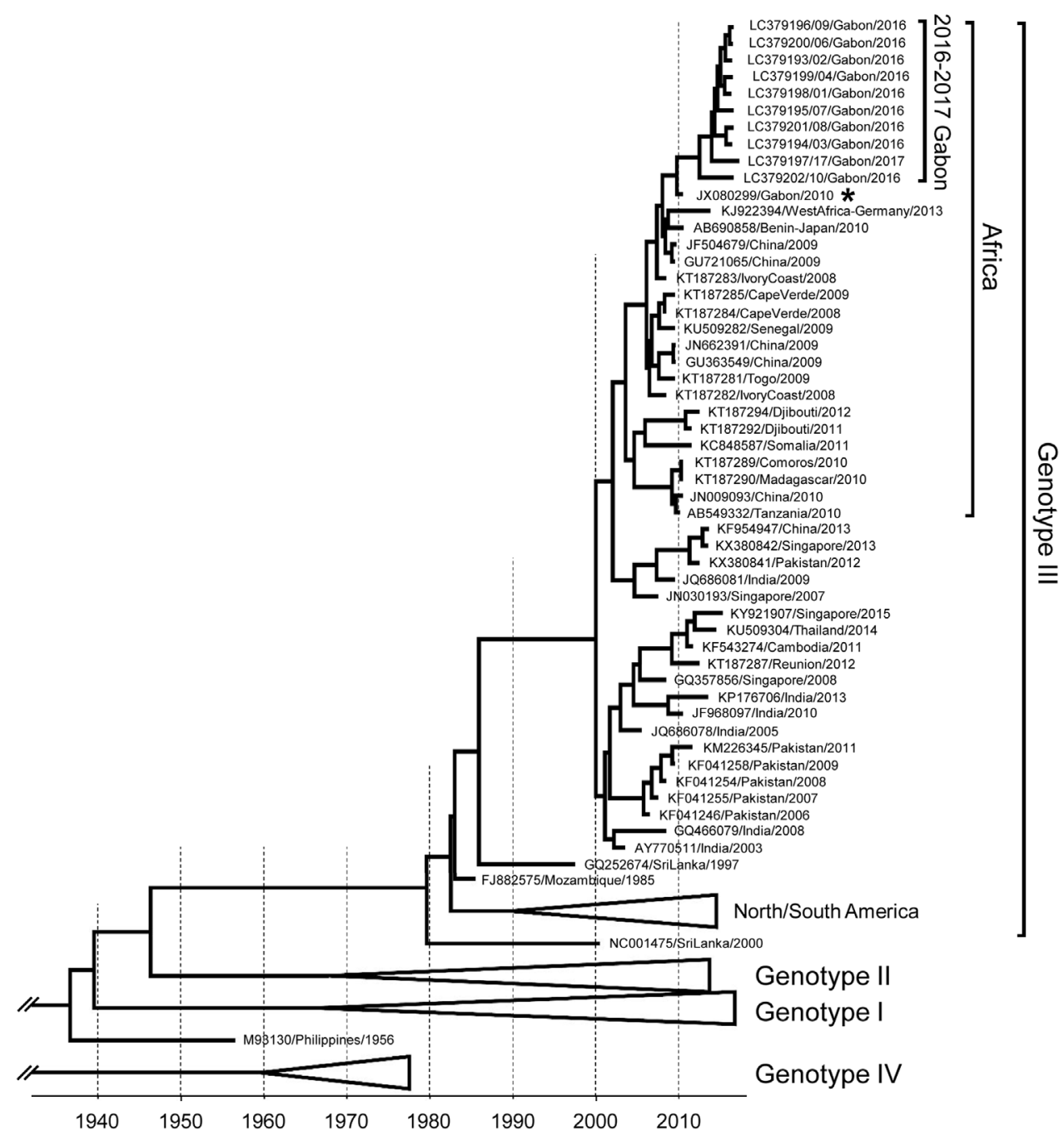

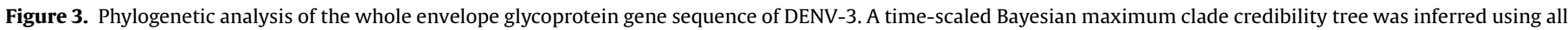

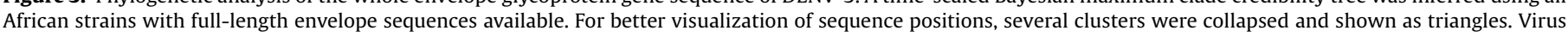

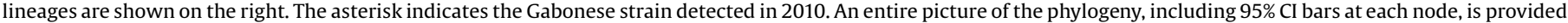
in the Supplementary material (Figure S1).

DENV-3 infection have been reported in Central Africa: Cameroon in 2006 (Domingo et al., 2011) and Gabon in 2010 (Caron et al., 2013). Both of the two Central African cases showed DENV-3 detection from only one individual; however, it is quite likely that DENV-3 infection cases have been underreported due to the unavailability of appropriate diagnostic tests in Africa (Amarasinghe et al., 2011). The latest report in Central Africa showed that $7.02 \%(8 / 114)$ of febrile patients in Douala, Cameroon were infected with DENV in 2017 (Yousseu et al., 2018), suggesting a similar infection rate of DENV in adults in Gabon (8.06\%, 15/186 in this study) and also, presumably, in neighboring Central African countries.

Unlike Southeast Asian countries, pediatric samples showed lower detection rates than adults in the present study, requiring future virological characterization of Gabonese DENV-3 for the possibility of preferential infection in adults and the identification of the mosquito vectors around the study area. The surveillance of symptomatic patients can provide information on circulating DENV strains, but may possibly miss approximately $65-67 \%$ of DENV-infected asymptomatic patients (Fritzell et al., 2018; Salje et al., 2018). A combination of molecular genetic data with epidemiological seroprevalence would be informative for understanding the whole context of DENV infections.

In Africa, DENV-3 has mainly been reported in West and East Africa, and in islands of the southeastern region (Virus Pathogen Resource, https://www.viprbrc.org/). African DENV-3 was first detected in Mozambique in 1985 (Gubler et al., 1986) and has been detected independently in East Africa since 1993 (e.g. Djibouti, Somalia, Tanzania, Madagascar) and in West Africa since 2006 (e.g. Senegal, Côte d'Ivoire, Togo, Benin). Phylogenetic studies showed that these occurrences of African DENV-3 infections were caused by viruses that originated from the Indian sub-continent (Messer et al., 2003). The time-scaled phylogenetic analysis in the present study revealed that the West African strains were likely transmitted into Gabon via Côte d'Ivoire, Togo, and Benin around 2008, before the first detection of DENV-3 in Gabon in 2010 (Figure 5). The phylogenetic tree also clarified that Gabonese DENV-3 strains were derived from a lineage distinct from the one that caused outbreaks in Cape Verde and Senegal in 2009.

In recent years, dengue caused by DENV-3 infection has mainly been reported in Sub-Saharan Africa in sentinel cases involving febrile returning travelers (Shihada et al., 2017). Interestingly, BLAST analysis of the complete DENV-3 genome sequenced in this study 


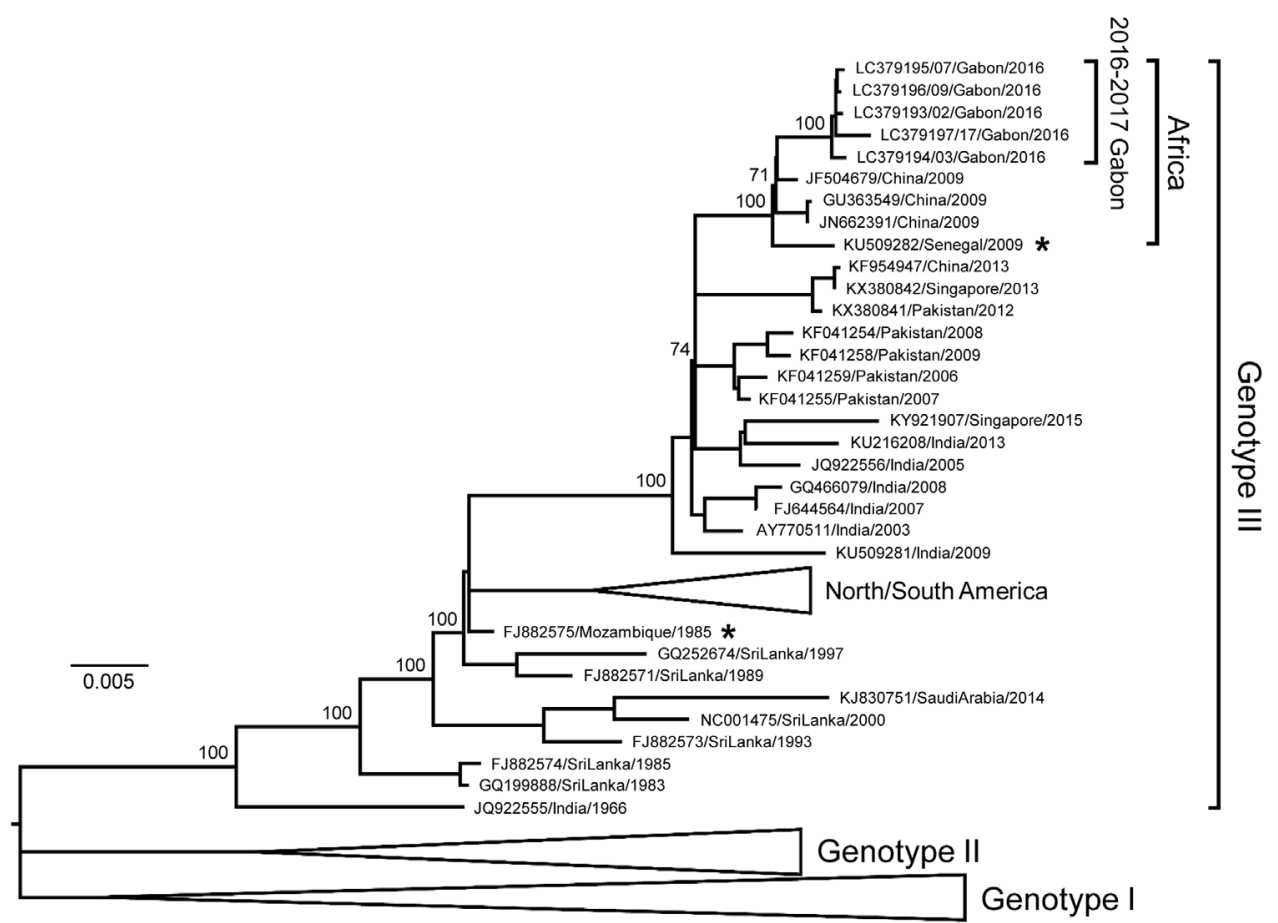

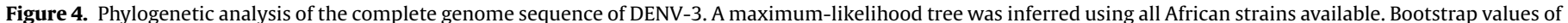

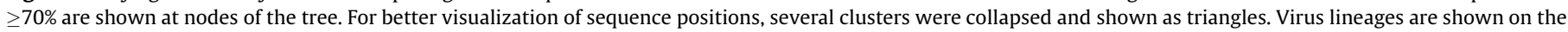

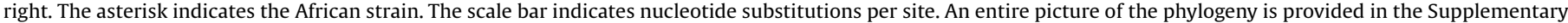
material (Figure S2).

Table 4

Amino acid substitutions in the whole polyprotein sequences of the Senegal 2009 strain and the strains sequenced in this study.

\begin{tabular}{|c|c|c|c|c|c|c|c|}
\hline \multirow[t]{2}{*}{ Genes } & \multirow[t]{2}{*}{ Amino acid position ${ }^{\mathrm{a}}$} & \multirow{2}{*}{$\begin{array}{l}\text { Reference } \\
\text { Senegal } 2009\end{array}$} & \multicolumn{5}{|c|}{ Gabon $^{\mathrm{b}}$} \\
\hline & & & $\begin{array}{l}\text { \#02 } \\
2016\end{array}$ & $\begin{array}{l}\# 03 \\
2016\end{array}$ & $\begin{array}{l}\# 07 \\
2016\end{array}$ & $\begin{array}{l}\text { \#09 } \\
2016\end{array}$ & $\begin{array}{l}\text { \#17 } \\
2017\end{array}$ \\
\hline$C$ & 93 & $S$ & $*$ & $*$ & $*$ & $*$ & G \\
\hline PrM & 171 & $\mathrm{~T}$ & $*$ & $*$ & A & A & $*$ \\
\hline PrM & 254 & $\mathrm{~L}$ & $*$ & $*$ & $*$ & $*$ & I \\
\hline $\mathrm{E}$ & 508 & $\mathrm{~T}$ & I & I & I & I & I \\
\hline $\mathrm{E}$ & 546 & $\mathrm{~T}$ & * & * & A & $*$ & $*$ \\
\hline $\mathrm{E}$ & 724 & A & $*$ & $*$ & V & $*$ & $*$ \\
\hline NS1 & 813 & $\mathrm{R}$ & $\mathrm{K}$ & $\mathrm{K}$ & $\mathrm{K}$ & $\mathrm{K}$ & $\mathrm{K}$ \\
\hline NS1 & 955 & $\mathrm{R}$ & $\mathrm{K}$ & $*$ & $*$ & $*$ & $*$ \\
\hline NS1 & 1101 & $\mathrm{D}$ & G & G & G & G & G \\
\hline NS2A & 1144 & $\mathrm{~L}$ & $\mathrm{~F}$ & $\mathrm{~F}$ & $\mathrm{~F}$ & $\mathrm{~F}$ & $\mathrm{~F}$ \\
\hline NS3 & 1682 & V & I & I & I & I & I \\
\hline NS3 & 1731 & V & I & I & I & I & I \\
\hline NS3 & 1943 & $\mathrm{~N}$ & $*$ & $*$ & $*$ & $*$ & $\mathrm{H}$ \\
\hline NS4A & 2155 & $\mathrm{~L}$ & * & * & * & * & $\mathrm{S}$ \\
\hline NS4B & 2353 & A & V & V & V & V & V \\
\hline NS5 & 2520 & K & $\mathrm{R}$ & $\mathrm{R}$ & $\mathrm{R}$ & $\mathrm{R}$ & $\mathrm{R}$ \\
\hline NS5 & 2764 & $\mathrm{D}$ & $*$ & $*$ & $*$ & $*$ & $\mathrm{~N}$ \\
\hline NS5 & 2784 & V & D & D & D & D & $\mathrm{D}$ \\
\hline NS5 & 2791 & K & $\mathrm{T}$ & $\mathrm{T}$ & $\mathrm{T}$ & $\mathrm{T}$ & $\mathrm{T}$ \\
\hline NS5 & 2796 & $\mathrm{R}$ & G & G & G & G & G \\
\hline NS5 & 2913 & A & $*$ & $*$ & $*$ & $*$ & V \\
\hline NS5 & 2978 & $P$ & $\mathrm{~L}$ & $\mathrm{~L}$ & $\mathrm{~L}$ & $\mathrm{~L}$ & $\mathrm{~L}$ \\
\hline NS5 & 3166 & $\mathrm{D}$ & $\mathrm{N}$ & $\mathrm{N}$ & $\mathrm{N}$ & $\mathrm{N}$ & $\mathrm{N}$ \\
\hline NS5 & 3240 & $\mathrm{E}$ & $*$ & D & $*$ & $*$ & $*$ \\
\hline NS5 & 3354 & $\mathrm{~S}$ & $\mathrm{P}$ & $\mathrm{P}$ & $\mathrm{P}$ & $\mathrm{P}$ & $\mathrm{P}$ \\
\hline
\end{tabular}

C, capsid; M, membrane; E, envelope.

a Number depicts the amino acid position in the polyprotein of the Senegal 2009 strain.

b Asterisk shows identical amino acid to the Senegal 2009 strain. identified Chinese DENV-3 strains isolated in 2009 in Zhejiang and Guangzhou as highly homologous strains (GenBank accession numbers JF504679, GU363549, and JN662391). However, these African-like Chinese strains were supposedly not maintained in China, because other recent Chinese strains isolated after 2012 were composed of Asian circulating strains in genotype II (Figures 3 and 4 ). These results may be indicative of international transmission and a sporadic spread of infections due to returning Chinese travelers or workers importing African DENV-3 strains. Similar to China, around 2009, a variety of European countries and Japan reported imported cases of DENV-3 strains belonging to genotype III from West Africa, possibly reflecting a large epidemic of DENV-3 in West Africa at that time (e.g. Moi et al., 2010; Ninove et al., 2009).

Epidemiological studies have suggested that severe symptoms might occur during secondary DENV infections with a different serotype (Guzman and Harris, 2015). Recently, two clinical studies demonstrated that a specific range of pre-existing DENV antibody titers were associated with the highest risk of severe symptoms, supporting the theory of antibody-dependent enhancement (ADE) of DENV infections (Katzelnick et al., 2017; Salje et al., 2018). The present study revealed a high anti-DENV IgG detection rate in DENV-3-positive patients with the risk of repeated DENV infections in Gabon, which is related to a report of anti-DENV seropositivity before the 2007 outbreak in Gabon (Gabor et al., 2016). Although a higher IgG titer correlated with a lower viral titer in this study, which might show an effective suppression of viral replication (Figure 2), the ELISA results showed that physicians need to pay attention to the occurrence of severe dengue in the country.

In conclusion, the present DENV surveillance conducted in febrile populations revealed the latest situation of DENV circulation in 


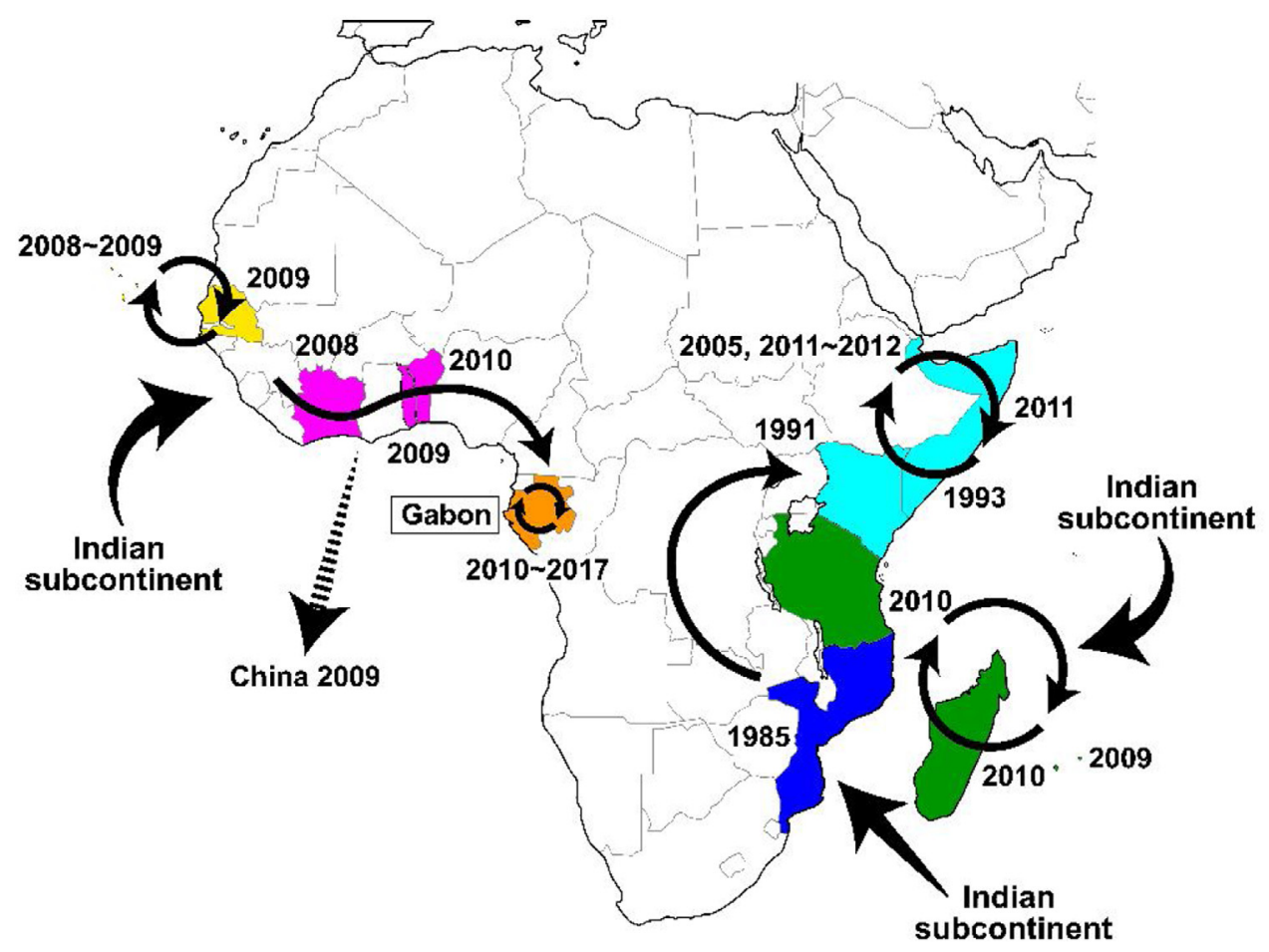

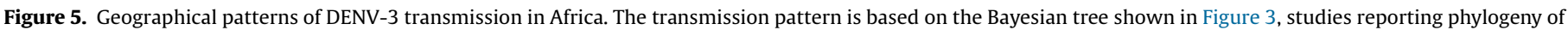

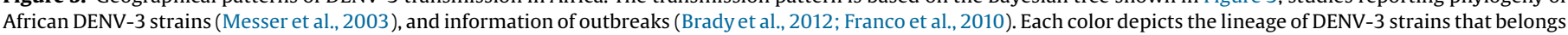
to a single cluster. Years show the time point of DENV-3 detection in the country.

Gabon, with a tendency to infection in adults rather than the pediatric population, and with peaks during the rainy seasons. The dissemination pattern of DENV-3 from West Africa to Gabon was also clarified by the phylogenetic tree. These findings will be informative in suggesting countermeasures for the spread of DENV in Gabon. However, it will be necessary to conduct continuous DENV surveillance to understand the current situation in order to help in the immediate alert and control of DENV infections.

\section{Funding sources}

This work was supported by the Science and Technology Research Partnership for Sustainable Development (SATREPS), Japan International Cooperation Agency (JICA) and the Japan Agency for Medical Research and Development (AMED), JP19jm0110013h; Grant-in-Aid for Scientific Research (KAKENHI), Japan Society for the Promotion of Science (JSPS), 17K15830.

\section{Ethical approval}

This study was approved by the Institutional Review Boards of CERMEL and Nagasaki University (approval numbers CEI-007 and 170921177, respectively). Written informed consent was obtained from all participants or their parents.

\section{Conflict of interest}

All authors have no conflicts of interest to declare.

\section{Acknowledgements}

We thank Mayuko Kimura, Sayaka Okada, and Maki Sueyoshi for technical assistance with the analyses at Nagasaki University, Miku Takano for management of logistics, and the entire staff at
CERMEL for their kind hospitality during the stay of the Japanese researchers.

\section{Appendix B. Supplementary data}

Supplementary material related to this article can be found, in the online version, at doi:https://doi.org/10.1016/j.ijid.2019.12.002.

\section{References}

Amarasinghe A, Kuritsk JN, Letson GW, Margolis HS. Dengue virus infection in Africa. Emerg Infect Dis 2011;17:1349-54.

Bhatt S, Gething PW, Brady OJ, Messina JP, Farlow AW, Moyes CL, et al. The global distribution and burden of dengue. Nature 2013;496:504-7.

Brady OJ, Gething PW, Bhatt S, Messina JP, Brownstein JS, Hoen AG, et al. Refining the global spatial limits of dengue virus transmission by evidence-based consensus. PLoS Negl Trop Dis 2012;6(8):e1760.

Caron M, Paupy C, Grard G, Becquart P, Mombo I, Nso BB, et al. Recent introduction and rapid dissemination of Chikungunya virus and Dengue virus serotype 2 associated with human and mosquito coinfections in Gabon, Central Africa. Clin Infect Dis 2012;55:e45-53.

Caron M, Grard G, Paupy C, Mombo IM, Bikie Bi Nso B, Kassa Kassa FR, et al. First evidence of simultaneous circulation of three different dengue virus serotypes in Africa. PLoS One 2013;8:e78030.

Domingo C, Niedrig M, Gascón J, Palacios G, Reyes N, Malo MJ, et al. Molecular surveillance of circulating dengue genotypes through European travelers. J Travel Med 2011;18:183-90.

Franco L, Di Caro A, Carletti F, Vapalahti O, Renaudat C, Zeller H, et al. Recent expansion of dengue virus serotype 3 in West Africa. Euro Surveill 2010;15(7) pii $=19490$

Fritzell C, Rousset D, Adde A, Kazanji M, Van Kerkhove MD, Flamand C. Current challenges and implications for dengue, chikungunya and Zika seroprevalence studies worldwide: a scoping review. PLoS Negl Trop Dis 2018;12:e0006533.

Gabor JJ, Schwarz NG, Esen M, Kremsner PG, Grobusch MP. Dengue and chikungunya seroprevalence in Gabonese infants prior to major outbreaks in 2007 and 2010: a sero-epidemiological study. Travel Med Infect Dis 2016;14:26-31.

Gubler DJ, Sather GE, Kuno G, Cabral JR. Dengue 3 virus transmission in Africa. Am J Trop Med Hyg 1986;35(6):1280-4.

Guzman MG, Harris E. Dengue. Lancet 2015;385(9966):453-65.

Katzelnick LC, Gresh L, Halloran ME, Mercado JC, Kuan G, Gordon A, et al. Antibodydependent enhancement of severe dengue disease in humans. Science 2017;358 (6365):929-32. 
Messer WB, Gubler DJ, Harris E, Sivananthan K, De Silva AM. Emergence and global spread of dengue serotype 3, Subtype III virus. Emerg Infect Dis 2003;9(7):800-9.

Moi ML, Takasaki T, Kotaki A, Tajima S, Lim CK, Sakamoto M, et al. Importation of dengue virus type 3 to Japan from Tanzania and Côte d'Ivoire. Emerg Infect Dis 2010;16(11):1770-2.

Ninove L, Parola P, Baronti C, De Lamballerie X, Gautret P, Doudier B, et al. Dengue virus type 3 infection in traveler returning from West Africa. Emerg Infect Dis 2009;15(11):1871-2.

Nisalak A, Endy TP, Nimmannitya S, Kalayanarooj S, Thisayakorn U, Scott RM, et al. Serotype-specific dengue virus circulation and dengue disease in Bangkok, Thailand from 1973 to 1999. Am J Trop Med Hyg 2003;68(2):191-202.

Pourrut X, Nkoghé D, Gonzalez JP, Leroy E. No evidence of dengue virus circulation in rural Gabon. Emerg Infect Dis 2011;17(8):1568-9.

Salje H, Cummings DAT, Rodriguez-Barraquer I, Katzelnick LC, Lessler J, Klungthong C, et al. Reconstruction of antibody dynamics and infection histories to evaluate dengue risk. Nature 2018;557:719-23.

Santiago GA, Vergne E, Quiles Y, Cosme J, Vazquez J, Medina JF, et al. Analytical and clinical performance of the CDC real time RT-PCR assay for detection and typing of dengue virus. PLoS Negl Trop Dis 2013;11:e2311.
Shepard DS, Undurraga EA, Halasa YA, Stanaway JD. The global economic burden of dengue: a systematic analysis. Lancet Infect Dis 2016;16(8):935-41.

Shihada S, Emmerich P, Thomé-Bolduan C, Jansen S, Günther S, Frank C, et al Genetic diversity and new lineages of dengue virus serotypes 3 and 4 in returning travelers, Germany, 2006-2015. Emerg Infect Dis 2017;23:272-5.

Simmons CP, Farrar JJ, Nguyen VV, Wills B. Dengue. N Engl J Med 2012;366 (15):1423-32.

Soo KM, Khalid B, Ching SM, Chee HY. Meta-analysis of dengue severity during infection by different dengue virus serotypes in primary and secondary infections. PLoS One 2016;11(5)e0154760.

Yousseu FBS, Nemg FBS, Ngouanet SA, Mekanda FMO, Demanou M. Detection and serotyping of dengue viruses in febrile patients consulting at the New-Bell District Hospital in Douala, Cameroon. PLoS One 2018;13:e0204143.

World Health Organization. Dengue increase likely during rainy season: WHO warns. 2019. . . [Accessed 20 September 2019] https://www.who.int/westernpacific/news/detail/11-06-2019-dengue-increase-likely-during-rainy-seasonwho-warns. 